\title{
Filières agro-industrielles et développement : le cas de la viande bovine dans les pays sahéliens*
}

\author{
PHILIPPE LEBAILLY \\ Faculté des Sciences Agronomiques de l'État, \\ Gembloux, Belgique
}

\begin{abstract}
RÉSUMÉ
L'étude des filières agro-alimentaires permet une approche intégrée des problèmes liés au développement des productions vivrières dans les pays du Tiers Monde. L'auteur de cet article, après avoir précisé les concepts en relation avec la notion de filière, livre ses réflexions à propos de l' installation de systèmes agro-industriels performants dans les pays en développement. Il expose ensuite succinctement les caractéristiques des différents maillons constitutifs de la filière de la viande dans les pays sahéliens. Il conclut que les États devraient impérativement favoriser l'éclosion de filières vivrières capables d'assurer un lien entre les producteurs et les consommateurs ainsi qu'un développement équilibré de la production, de la commercialisation et des débouchés.
\end{abstract}

\begin{abstract}
The study of the food subsector permits an integrated approach to the problems linked to the development of food production in Third World countries. The author, after defining various concepts related to the subsector notion, explains how successful agro-industrial systems can be developed in Third World countries and outlines the various steps in the meat production network of Sahelian countries. Food subsectors, he concludes, create a link between producers and consumers and, as such, are instrumental in ensuring a balance between production, commercialization and markets.
\end{abstract}

\section{INTRODUCTION}

Ces dernières années, l'accent a été mis sur l'intérêt d'une intégration des disciplines et d'une prise en compte des interactions entre la production agricole et sa valorisation.

L'approche en terme de filière semble particulièrement de mise dans les pays en développement où il importe de tirer le meilleur parti possible des facteurs productifs. Les

* Première version reçue le 7-12-87; version définitive acceptée le 9-08-88. 
systèmes de production se doivent d'être compris, analysés et quantifiés afin d'être renforcés.

L'objectif de cet article est d'insister sur la nécessité d'installer prioritairement des filières vivrières performantes dans les pays en développement.

Nous décrirons succinctement les maillons constitutifs d'une filière à partir de l'exemple de la viande dans les pays sahéliens. En bref, trois points essentiels seront abordés :

- la définition des concepts;

- quelques réflexions sur les filières agro-alimentaires dans les pays en développement;

- l'exemple de la filière de la viande.

\section{CONCEPTS}

Le vocable "filière ", connaît depuis quelques années une vogue singulière en économie. Les politiques, les plans, les programmes de développement s'y réfèrent volontiers aujourd'hui et s'établissent, le plus souvent, en terme de filière (ou mieux sous l'alibi de la filière) pour avoir droit de cité.

Néanmoins, la notion n'est pas nouvelle. Dès le 18ème siècle, certaines actions des pouvoirs publics auraient été guidées par une approche faisant intervenir les filières (Stoffaes, 1980) et, tout en ne recourant pas au terme de filières, des géographes et des sociologues ont décrit, depuis le début du siècle, certaines filières de produits agricoles.

La théorie des filières s'est essentiellement développée parmi les économistes francophones (Sekkat, 1987). Du côté anglo-saxon, James Shaffer se fit, à partir de 1968, l'avocat de l'intérêt de l'analyse des filières en économie rurale (Shaffer, 1968 et 1973).

Chacun dispose de sa propre définition et des limites qu'il entend donner au mot « filière ». Nous voudrions en conséquence proposer (il n'y a pas en la matière de vérité transcendante) au préalable une définition stricte du terme et de sa portée.

La filière désigne l'ensemble des actes de production, transformation, distribution relatifs à un produit (pomme de terre, sucre, bois) ou à un groupe de produits homogènes (céréales, fruits, légumes, viandes de boucherie, produits laitiers, ...) et concourant à la satisfaction d'un même besoin final issu de la consommation (Ledent, 1986). La filière représente en conséquence l'itinéraire d'un produit, son cheminement. Elle comprend des transformations physiques visibles et des interventions extérieures moins transparentes qui modifient certaines caractéristiques essentielles dudit produit.

Notre réflexion portera uniquement sur les filières-produits qui correspondent à une vision technique du concept. Les différentes filières pour lesquelles le critère d'appartenance repose sur le concours des différents maillons à la satisfaction d'une demande finale (filière transport, filière énergie, filière loisir, ...) ne seront pas abordées dans le cadre de cette recherche. Elles ont été largement discutées d'un point de vue théorique par différents auteurs (French, 1974; Monfort, 1983; Sekkat, 1986 et Tollet, 1982).

Un des problèmes qui restent posés est de déterminer à quel stade s'arrête l'amont et/ou l'aval d'une filière, quels opérateurs appartiennent ou non à une filière produit. La réponse est délicate. L'appartenance à une filière pourrait se résumer à la question de savoir si le maillon considéré ne peut exister, tel quel, que grâce aux relations établies avec le système. En aval, nous nous réfèrerons plus volontiers au concept lié à la perte de signification du produit (Fourichon, 1986). Le plus souvent, les objectifs poursuivis privilégient 
une vision verticale des filières étudiées ( « du producteur à l'assiette du consommateur ») qui devront impérativement et en permanence être restituées dans le contexte plus général du complexe agro-alimentaire (voire agro-industriel).

Parallèlement, mais non accessoirement, au concept de la filière, il convient d'introduire la notion d'intégration, qui, au sens général du terme, désigne la réunion d'une série d'entreprises, au départ indépendantes, dans un ensemble lié par l'autorité d'un centre de décision fréquemment appelé « pôle d'intégration ». Selon que ce dernier, coordonne des maillons successifs ou identiques d'une filière-produit, nous parlerons d'intégration verticale ou horizontale.

L'intégration verticale a été analysée par divers auteurs (Casson, 1984 et Kilmer, 1986). Les avantages ou inconvénients de celle-ci pour les différents opérateurs de la filière (essentiellement le pôle d'intégration et les producteurs agricoles) ont été largement discutés. Parmi ceux-ci, nous citerons :

- l'apparition de vastes ateliers de production qui résultent soit de l'accroissement de la taille des exploitations, soit de la juxtaposition d'une agriculture industrielle hautement capitalisée et d'une agriculture paysanne dont la structure est essentiellement familiale;

- la spécialisation des productions et des tâches (division du travail);

- la possibilité de pouvoir écouler sa production avec une certaine garantie de prix et de revenu;

- l'abandon du pouvoir de décision de l'exploitant agricole qui se transforme en véritable salarié;

- la contribution du pôle intégrateur au financement externe de l'agriculture et au problème du crédit qui se pose le plus souvent aux exploitants agricoles;

- le développement de l'encadrement technique.

L'intégration horizontale, dont l'entraide et la coopération sont les formes les plus fréquemment rencontrées au niveau de filières agro-industrielles, constitue une réponse à l'intégration verticale. Les opérateurs d'un même maillon s'unissent au sein de groupements ou de coopératives afin d'accroître leur poids face au pôle intégrateur.

L'approche en terme de filière permet également, en systématisant les relations de vendeurs et d'acheteurs, d'étudier les modes de coordination entre la demande et l'offre à chaque étape.

Enfin, intégrant les données technico-économiques des maillons qui la composent, les filières-produits sont un préalable à la compréhension d'un processus de production. Néanmoins, sous cet angle, elles s'avèrent peu opérationnelles pour prévoir les transformations d'un système soumis à des facteurs de changements. Seul le passage méthodologique de la notion de filière-produit à la notion de filière de la demande finale étend la capacité explicative et prévisionnelle du concept (Tollet, 1982).

\section{LES FILIÈRES AGRO-ALIMENTAIRES DANS LES PAYS EN DÉVELOPPEMENT}

Dans la plupart des pays en développement, force est de constater que nous sommes schématiquement en présence de deux types de filières. Les premières sont généralement intégrées verticalement et concernent essentiellement la production de biens destinés à l'exportation communément appellés «produits de rente ». Les secondes sont atrophiées 
ou complexes et mal connues. Elles regroupent les produits vivriers destinés à couvrir les besoins locaux.

En Afrique, par exemple, où des produits de base tel que le manioc, le mil, le maïs, le sorgho, les haricots secs, etc. revêtent une importance particulière, les techniques de mouture et de transformation des produits vivriers restent ancestrales et relèvent des pratiques anciennes. À l'aide de mortiers ou pilons, les paysannes représentent les opérateurs principaux de la transformation, voire des activités de la filière vivrière. En effet, dans beaucoup de zones en Afrique, les cultures vivrières sont l'apanage de la femme qui, lorsqu'un surplus est produit, écoule encore ce dernier sur les marchés locaux.

Les industries transformatrices produisant farines et semoules grâce à des approvisionnements et susceptibles d'écouler une partie de la production vers les grands centres urbains ne sont pas légion dans les pays en développement. Tout au plus, l'activité des entrepreneurs locaux et les capitaux étrangers se sont concentrés dans les zones portuaires vers les activités liées à la transformation des céréales importées ou reçues sous forme d'aide. L'existence de ces infrastructures concurrence aujourd'hui les initiatives qui seraient prises dans les zones de production vivrière et contribue au changement des habitudes de consommation dans les villes.

Aux côtés des filières vivrières dont le développement est en quelque sorte bloqué, se sont installées de véritables enclaves agro-industrielles (Chataigner, 1982), travaillant directement pour le marché mondial (produits de rente), organisant et contrôlant, dans un espace donné, tous les maillons du processus de production depuis l'approvisionnement en intrants jusqu'à l'expédition du produit fini. Les capitaux, le savoir-faire et l'encadrement sont importés.

La majorité des projets de développement agricole étaient articulés et s'articulent encore sur ce schéma, même lorsque l'objectif premier vise le développement rural intégré ${ }^{1}$ d'une région.

L'argument, le plus souvent présenté pour justifier ces systèmes d'exploitation tournés vers les exportations agricoles est celui du sous-emploi des ressources lorsque l'exploitant agricole satisfait seulement les besoins locaux. Il remonte à A. Smith («Vent for surplus »).

Aujourd'hui, les problèmes liés à la préservation du capital foncier et à la rareté croissante des terres cultivables contraignent les paysans, les entrepreneurs agricoles et les pouvoirs publics à faire des choix entre la production de rente et la production alimentaire.

Les analyses théoriques et les enseignements des expériences menées dans ce domaine mènent à des résultats et des conclusions divergents : certains prônent la promotion des cultures d'exportation, seules capables de stimuler les productions vivrières, d'autres estiment que la promotion des exportations a des répercussions néfastes sur le secteur vivrier.

Nous ne trancherons évidemment pas la question et nous nous limiterons à reprendre le constat fait par Basler (1986) sur les systèmes d'organisation en Afrique :

1. « Le développement rural intégré est une stratégie par laquelle une série d'actions régulières et progressives, annoncées ou soutenues par une volonté politique, apporte des changements quantitatifs et qualitatifs au sein d'une population rurale et avec sa participation consciente et active, en vue de répondre à ses besoins essentiels, d'améliorer son bien-être et d'engendrer un processus autonome de développement » — EIB, Développement rural intégré, Fascicule $n^{\circ} 2$ : L'approche pratique, conclusions des travaux du séminaire organisé à Bordeaux du 16 juin au 11 juillet 1980, Talence, 1980, 76p. 
- le marché des intrants est largement orienté vers les cultures de rente; dans les zones tournées exclusivement vers les cultures vivrières, la distribution des intrants agricoles fait le plus souvent défaut;

- les crédits de campagne sont octroyés pour les cultures de rente qui sont commercialisées et dont une partie des recettes servira au remboursement du prêt; en revanche, les produits de base récoltés sont essentiellement autoconsommés, le surplus est écoulé le plus souvent à vil prix et le contrôle des recettes échappent à l'organisme de crédit;

- l'infrastructure routière a été mise en place prioritairement selon les axes utilisés pour l'exportation, les zones rurales productrices de denrées vivrières ont été très souvent délaissées;

- les progrès réalisés par la recherche sont plus difficilement diffusés lorsqu'ils concernent les cultures vivrières, la recherche appliquée et les services de vulgarisation faisant généralement défaut.

Ce diagnostic nous montre que seule la mise en place de filières vivrières dans les pays en développement contribuera à une intensification de la production et à une stabilité des circuits de commercialisation.

Les États se doivent, nous semble-t-il, d'œuvrer prioritairement dans ce sens en favorisant l'éclosion de maillons en amont et en aval de l'agriculture vivrière. Il y aurait ainsi un déplacement de la valeur ajoutée en amont et en aval de l'exploitation agricole qui permettrait in fine d'accroître la productivité et le revenu des paysans.

Pour ce faire, les missions des États devraient être appelées à se transformer. Tout en poursuivant leur rôle de catalyseur et de régulateur de l'économie, les États s'emploieront à se doter d'infrastructures aptes à susciter le développement économique. Parallèlement, des moyens seront mis en œuvre pour stimuler l'initiative privée afin que de petites et moyennes entreprises se créent, prennent le relai et assurent un nouvel essor au développement tout en désengorgeant l'hypertrophie des structures administratives.

\section{L'EXEMPLE DE LA FILIÈRE DE LA VIANDE}

\section{A. INTRODUCTION}

Certains pays en développement (les pays sahéliens notamment) possèdent un potentiel de production animale élevé et des perspectives d'expansion de la commercialisation du bétail et des produits de l'élevage existent (tableau 1). Ces pays accordent fort logiquement une place prioritaire aux programmes visant une meilleure valorisation des ressources animales nationales, voire le développement des exportations vers des pays tiers. Globalement, l'essor des activités liés à l'élevage devrait permettre une élévation du revenu agricole et favoriser le renforcement de la production primaire et des activités de commercialisation. Nous soulignerons qu'une partie importante de la production animale des pays sahéliens provient de régions dans lesquelles les autres emplois possibles des ressources de main-d'œuvre et de terre sont extrêmement limités.

Sous peine de provoquer un gaspillage des ressources, les plans visant toute modification importante dans le secteur de la commercialisation ou de la production doivent être évalués par rapport à l'ensemble du système. La commercialisation ${ }^{2}$ du bétail et de

2. La commercialisation désigne toutes les opérations intervenant entre l'achat de l'animal au producteur primaire (naisseur) et la vente finale de la viande et des abats au consommateur. 
Tableau 1

Caractéristiques de l'élevage dans les principales régions d'Afrique tropicale

\begin{tabular}{|c|c|c|c|c|c|c|c|c|c|c|c|c|}
\hline \multirow{2}{*}{ RÉGIONS } & \multicolumn{2}{|c|}{ Superficie des terres } & \multicolumn{2}{|c|}{$\begin{array}{c}\text { Superficie des prairies } \\
\text { et pâturages }\end{array}$} & \multicolumn{3}{|c|}{ Population agricole } & \multicolumn{2}{|c|}{ UBT (5) } & \multirow{2}{*}{$\begin{array}{c}\begin{array}{c}\mathrm{UBT} / \\
\mathrm{km}\end{array} \\
(\mathrm{C} / \mathrm{A})\end{array}$} & \multirow{2}{*}{$\begin{array}{c}\begin{array}{c}\text { UBT/popul. } \\
\text { agricole }\end{array} \\
(\mathrm{C} / \mathrm{B})\end{array}$} & \multirow{2}{*}{$\begin{array}{c}\begin{array}{c}\mathrm{UBT} / \mathrm{km} \\
\text { de prairies }\end{array} \\
\mathrm{C} / \mathrm{D}\end{array}$} \\
\hline & $\begin{array}{c}(1000 \mathrm{~km}) \\
\mathrm{A}\end{array}$ & $\%$ du total & $\begin{array}{c}(1000 \mathrm{~km}) \\
\mathrm{D}\end{array}$ & $\%$ du total & $\begin{array}{c}\text { (1000 hab.) } \\
\text { B }\end{array}$ & $\%$ du total & $\mathrm{B} / \mathrm{A}$ & $\begin{array}{l}\text { (milliers) } \\
\mathrm{C}\end{array}$ & $\%$ & & & \\
\hline $\begin{array}{l}\text { Pays du Sahel } \\
\text { dont: } \\
\text { - Burkina-Faso } \\
\text { - Gambie } \\
\text { - Mali } \\
\text { - Mauritanie } \\
\text { - Niger } \\
\text { - Sénégal } \\
\text { - Soudan } \\
\text { - Tchad }\end{array}$ & $\begin{array}{r}7.628 \\
\\
274 \\
10 \\
1.220 \\
1.030 \\
1.267 \\
192 \\
2.376 \\
1.259\end{array}$ & 35 & $\begin{array}{r}1.953 \\
\\
100 \\
1 \\
300 \\
393 \\
92 \\
57 \\
560 \\
450\end{array}$ & 34 & $\begin{array}{r}14.355 \\
\\
6.082 \\
540 \\
6.893 \\
1.296 \\
5.604 \\
5.252 \\
14.355 \\
4.028\end{array}$ & 5 & 1,9 & $\begin{array}{r}39,719 \\
2.736 \\
242 \\
4.614 \\
1.787 \\
3.825 \\
1.877 \\
20.030 \\
4.608\end{array}$ & 28 & 5,2 & 2,8 & 20,3 \\
\hline $\begin{array}{l}\text { Autres pays de } \\
\text { l'Afrique de l'Ouest } \\
\text { dont: } \\
\text { - Nigéria } \\
\text { - autres (1 }\end{array}$ & $\begin{array}{r}2.066 \\
\\
911 \\
1.155\end{array}$ & 10 & $\begin{array}{l}345 \\
210 \\
135\end{array}$ & 6 & $\begin{array}{l}93.061 \\
\\
65.210 \\
27.851\end{array}$ & 33 & 45 & $\begin{array}{r}17.937 \\
12.485 \\
5.452\end{array}$ & 15 & 8,7 & 0,2 & 52,0 \\
\hline Afrique centrale (2) & 3.988 & 18 & 353 & 6 & 31.499 & 11 & 7,9 & 6.760 & 5 & 1,7 & 0,2 & 19,2 \\
\hline Afrique de l'Est (3) & 3.456 & 16 & 1.193 & 21 & 97.252 & 35 & 28,1 & 58.646 & 41 & 14,7 & 0,6 & 49,2 \\
\hline Afrique Australe (4) & 3.820 & 18 & 1.587 & 27 & 35.391 & 13 & 9,3 & 11,775 & 8 & 3,1 & 0,3 & 7,4 \\
\hline Madagascar & 581 & 3 & 340 & 6 & 8.076 & 3 & 13,9 & 7.523 & 5 & 12,9 & 0,9 & 22,1 \\
\hline $\begin{array}{l}\text { Total Afrique } \\
\text { tropicale }\end{array}$ & 21.539 & 100 & 5.771 & 100 & 279.634 & 100 & 13 & 142.360 & 100 & 6,6 & 0,5 & 24,7 \\
\hline
\end{tabular}

Source: FAO - annuaire de la production, 1986

(1) Bénin, Ghana, Guinée, Côte d'Ivoire, Libéria, Sierra Leone, Togo et Guinée-Bissau

(2) Cameroun, Congo, Gabon, Zaïre, RCA et Guinée Équatoriale

(3) Burundi, Djibouti, Éthiopie, Kenya, Rwanda, Somalie, Tanzanie et Ouganda

(4) Angola, Botswana, Mozambique, Zimbabwe et Zambie

(5) UBT (Unité de Bétail Tropical) correspond à un bovin de $250 \mathrm{~kg}$ vif

1 chameau $=1$ UBT; 1 bovin $=0,7$ UBT; 1 bovin ou caprin $=0,10$ UBT 
la viande permet d'illustrer l'intérêt d'une approche en terme de filière dans les pays en développement et nous étudierons plus spécialement la situation en Afrique sahélienne.

Nous avons fixé les limites du Sahel à huit pays (Burkina-Faso, Gambie, Mali, Mauritanie, Niger, Sénégal, Soudan et Tchad). Cette délimitation est critiquable. La zone sahélienne (dont les deux principaux critères de définition sont par ordre d'importance la quantité annuelle des pluies et la longueur de la saison sèche) ne couvre pas avec exactitude les frontières politiques. Des pays comme le Sénégal ou le Tchad sont en partie sahéliens. En outre, l'élevage sub-saharien est un élevage sans structures et mouvant. Il existe, en effet, des flux saisonniers de bétail importants et complexes qui s'étendent globalement du Nord (hivernage) vers le Sud (saison sèche). De plus, nous pouvons distinguer des mouvements orientés vers les centres de consommation que représentent les pays maritimes tels le Nigéria et la Côte d'Ivoire.

Afin de clarifier l'approche, nous nous sommes efforcés dans la mesure du possible de traiter indépendamment chacun des maillons constitutifs de la filière étudiée, qui doivent être eux-mêmes subdivisés en fonction des catégories d'animaux.

Dans le cadre de cet article, nous envisagerons essentiellement les caractéristiques de l'élevage bovin. Nous soulignerons néanmoins que, dans la plupart des pays sahéliens, les troupeaux sont composés d'un assemblage d'espèces (principalement bovins, ovins et caprins), l'idéal pour l'éleveur étant d'avoir un cheptel judicieusement équilibré pour répartir les risques en cas d'incident climatique ou de maladie. Conformément à l'organigramme général de la filière de la viande (figure 1) cinq points essentiels seront examinés : la production, la collecte, l'abattage, la transformation et la consommation. Enfin, et en guise de conclusion, nous envisagerons le rôle joué par l'État sur les différents maillons de la filière de la viande au Sahel.

\section{B. LA PRODUCTION}

Dans la plupart des pays sahéliens, les éleveurs de gros bovidés accordent un faible intérêt à la consommation de leurs animaux. Ils appartiennent à des ethnies nomades ou transhumantes qui élèvent essentiellement des bovidés pour leur propre subsistance. Une partie des produits de l'élevage est réinsérée dans le cheptel afin de perpétuer le processus de reproduction du troupeau, une autre est autoconsommée, une troisième est nécessaire à la satisfaction des besoins essentiels. Tous ces produits assurent la reproduction simple du système de production et la survie du groupe. Les surplus éventuels seront thésaurisés en vue d'une consommation sociale et/ou prélevés, en partie du moins, par l'État (paiement de l'impôt).

Les effets des grayes sécheresses qui ont culminé avec l'hivernage désastreux de 1984 ont eu sur l'élevage sahélien des conséquences importantes, à savoir : $a$ ) les effectifs du cheptel bovin ont subi des pertes sévères; $b$ ) certains éleveurs ont vendu à bas prix des animaux en provenance de leur noyau de base; $c$ ) les épizooties se sont développées (peste, charbon, brucellose).

Aujourd'hui, nous assistons à un repli psychologique des populations vers des attitudes de survie autarcique, un choix radical étant opéré en faveur de la recherche de la sécurité plutôt que de la prise de risques. L'accent est mis prioritairement sur la satisfaction des besoins fondamentaux et les revenus monétaires sont délaissés.

Aéanmoins, le développement de la production ne pourra s'opérer que si l'éleveur s'oriente vers la spécialisation et l'échange. Les résultats dépendront essentiellement de 
Figure 1

Organigramme de la filière

de la viande de boucherie

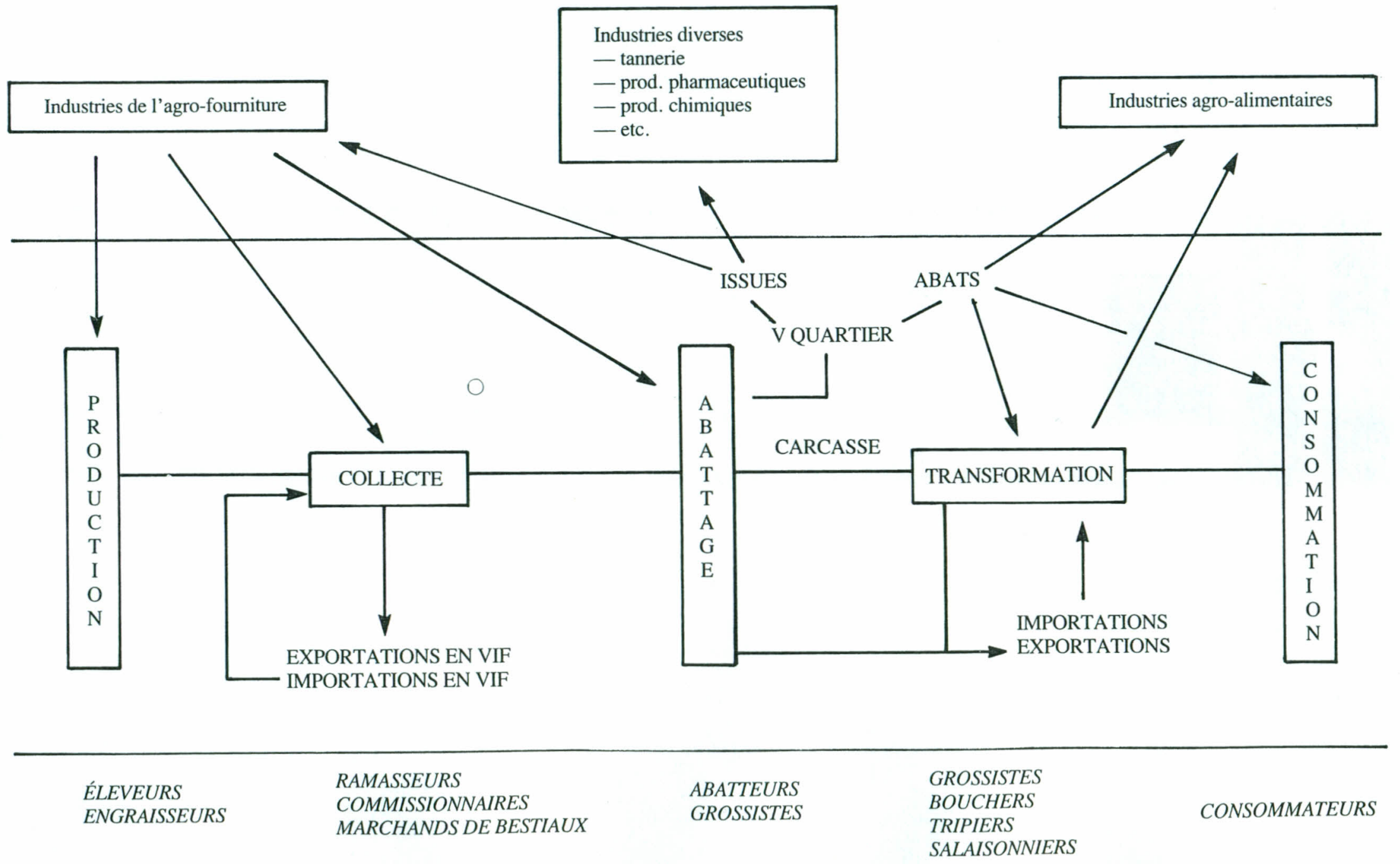


la capacité du producteur d'adapter sa production au besoin du marché, la production et le système de commercialisation ne pouvant en aucune façon devancer la demande.

Cela n'implique pas nécessairement une production à grande échelle mais un encadrement efficace sur le terrain afin de sensibiliser l'éleveur à l'emploi de méthodes plus productives, en conformité avec les besoins du marché.

Enfin, les systèmes de production « exportés », tels ceux qui dissocient dans l'espace les principaux stades de la production (naissance - élevage - engraissement), fréquemment pratiqués dans les pays développés, seront considérés avec une extrême précaution.

Ainsi, les projets d'embouche en milieu paysan se sont généralement soldés par des échecs parce qu'ils ne respectaient pas le cadre de l'exploitation familiale, la pratique d'un élevage bovin étant considérée comme avilissante par la plupart des cultivateurs.

\section{LA COLLECTE}

À ce stade, interviennent des opérateurs communément appelés «marchands de bestiaux » qui concentrent l'offre des différentes catégories de bétail au niveau du marché en vif. Dans les pays en développement, les marchés ruraux sont souvent de simples foires aux bestiaux équipées d'installations sommaires qui appartiennent aux pouvoirs locaux. Ceux-ci exercent un contrôle sur le marché et perçoivent une taxe (« droit de place ») qui, idéalement, ne devrait pas dépasser le coût des services fournis.

Par ailleurs, aux abords des centres urbains importants où les principaux mouvements du bétail confluent généralement au départ des zones d'élevage, nous observons la présence de vastes marchés centraux, plaques tournantes de la vente en gros et situés près d'un abattoir.

Dans tous les cas, les services et les installations des marchés (ruraux ou centraux) seront adaptés aux véritables besoins et aux desiderata des usagers et non pas conçus sur des modèles importés, dotés d'équipements complexes (stalles, ponts-bascules, caillebotis,...) coûteux et jamais utilisés.

Les marchands sont souvent accusés de faire des opérations spéculatives en achetant bon marché, en revendant cher et en alourdissant les prix à la consommation. Ils améliorent cependant la commercialisation en constituant des lots homogènes qui répondent aux besoins des acheteurs. Pour l'éleveur, le marchand apparait comme un maillon important dans la filière; une confiance réciproque est à la base de leurs échanges. Dans les pays sahéliens, les négociants locaux peuvent remplir parallèlement mais non accessoirement d'autres fonctions. Ils assurent, par exemple, l'approvisionnement des éleveurs en denrées alimentaires et en biens de consommation.

Enfin, la présence d'un marché accessible pour l'éleveur dans les zones rurales permet à ce dernier d'être renseigné sur les prix et les perspectives de vente.

\section{L'ABATtAGE}

L'abattoir, goulet d'étranglement de la filière des animaux de boucherie est l'outil clé dans le commerce de la viande. Sa taille, sa localisation, sa gestion influencent directement le bon fonctionnement et la dynamique du système et contribue à son efficience. Le problème de l'abattoir est complexe; la résolution des questions posées fait appel à une foule d'éléments d'information de nature diverse (économique, sanitaire, juridique, sociologique, etc.) et à maintes compétences. Dans les pays en développement, l'abattage 
à partir d'installations de fortune ou « au seuil même de l'étal du boucher » présente des inconvénients majeurs surtout d'ordre hygiénique et fiscal. Dans ce contexte, l'établissement d'abattage doit être considéré comme un service public offert aux abatteurs pour favoriser l'approvisionnement en concentrant l'offre et, dès lors, en facilitant également le contrôle sanitaire des viandes destinées à la consommation.

La localisation de l'abattoir dans les pays sahéliens se fera préférentiellement aux abords des grands centres de consommation. Cette situation se justifie par les infrastructures routières et ferroviaires généralement déficientes dans ces pays et par la préférence marquée des consommateurs pour la viande fraîchement abattue. En outre, l'établissement d'abattage doit s'intégrer dans l'ensemble du système de commercialisation, il ne sera pas uniquement le lieu où s'effectue l'abattage des animaux et le dépeçage des carcasses. Il convient également d'insister sur l'importance de la récupération du cinquième quartier ${ }^{3}$, spécialement des issues.

Bien entendu, dans les pays en développement, le volume des sous-produits non comestibles est très limité et, outre les peaux brutes, seules quelques parties de l'animal abattu ne sont pas commercialisées par le boucher. Ce sont essentiellement les contenus des panses, les cornes et onglons et le sang, sous-produits qui s'accumulent le plus souvent aux abords de l'abattoir ou sont rejetés à la rivière. La non récupération desdits produits provoquent des nuisances (odeurs, insectes et rongeurs) ou contribuent à la dissémination de germes pathogènes (charbon, brucellose,...).

Une solution consiste à transformer les issues en un engrais organique adapté aux cultures maraîchères qui ceinturent généralement les centres urbains. Le calcul économique de la rentabilité de l'opération devra impérativement prendre en compte les aspects sanitaires et de lutte contre les nuisances du projet. En effet, il semble tout à fait paradoxal de financer de vastes campagnes de vaccination alors que le sang des animaux abattus et les germes qu'il contient sont dirigés vers le fleuve en aval duquel des animaux viennent s'abreuver.

\section{E. LA TRANSFORMATION ET LA CONSOMMATION}

Dans les pays en développement, le système traditionnel de vente de la viande de boucherie est difficilement contournable. Les quantités consommées par habitant sont peu élevées. Les viandes de faible qualité, moins onéreuses, sont recherchées par les consommateurs au bas revenu.

Dans ces conditions, la découpe et la distribution de la viande sont aux mains de petites entreprises aux installations rudimentaires. Les bouchers locaux offrent un produit sans attrait, présenté sous la forme la plus simple. Les méthodes traditionnelles de vente sont hautement compétitives lorsqu'elles s'adressent à des populations au pouvoir d'achat peu élevé.

Les consommateurs plus aisés qui achètent des quantités plus importantes de viande devraient en principe rechercher des formes de distribution plus sophistiquées (boucheries où la qualité, la variété et les services seraient appréciables).

3. Par analogie aux quatre quartiers de la carcasse, on regroupe sous l'appellation « cinquième quartier ", les sous-produits provenant de la transformation d'un animal vivant en carcasse de boucherie, celleci étant constituée par les quatre quartiers de l'animal. Le cinquième quartier est un vaste ensemble représentant de 20 à $55 \%$ du poids de l'animal vivant et se divisant en deux parties : $a$ ) une partie comestible : les abats; $b$ ) une partie non comestible : les issues. 
Néanmoins, en Afrique, les ménagères qui disposent de revenus élevés font rarement le marché elles-mêmes et confient à des domestiques l'achat de la viande. Ceux-ci sont moins exigeants et peu enclins à rechercher une viande provenant de boucheries modernes.

En conséquence, la modernisation de ce maillon de la filière s'opérera avec une extrême prudence et sur base d'une étude du marché détaillée. Les investissements qui seraient réalisés à ce stade se doivent d'être rentabilisés par l'existence d'une demande ferme pour une viande de qualité.

\section{F. RÔLE JOUÉ PAR L'ÉTAT}

La problématique actuelle de la filière de la viande dans les pays sahéliens n'est pas simple. Elle exige la prise en compte de facteurs historiques qui ont été relatés avec beaucoup de justesse par Giri (1983).

En fait, la colonisation et l'avènement des jeunes républiques sahéliennes ont profondément modifié les rapports de force au sein des filières agro-alimentaires. Nous sommes passés d'un système régi harmonieusement par les sociétés traditionnelles à une organisation étatique qui, tout en recherchant le développement des productions animales par diverses actions, a toujours pris fait et cause pour le sédentaire (généralement cultivateur) dans les conflits l'opposant à l'éleveur transhumant.

Bien plus, les actions sanitaires menées par les services de l'élevage et la création de nouveaux points d'eau ont exacerbé les relations conflictuelles : sous l'effet des campagnes de vaccination et des nouvelles disponibilités en eau, le cheptel bovin s'est accru alors que les ressources fourragères stagnaient.

Face à cet accroissement des effectifs, les États ont voulu stimuler les exportations de viande vers les pays qui s'approvisionnaient traditionnellement au Sahel en animaux vivants. Ils ont mis en place des installations d'abattage performantes situées dans les capitales afin d'expédier les viandes fraîches à partir des aéroports.

Parallèlement, les niveaux des taxes sur les têtes d'animaux ont été élevés. La structure du cheptel s'est alors modifiée. Les animaux ont été abattus plus jeunes et le poids moyen à l'abattage a diminué. Le troupeau, qui traditionnellement dans les zones sahéliennes était constitué d'une part importante d'animaux âgés (et plus résistants) pour permettre en cas d'épidémies la reconstitution du noyau de base, s'est rajeuni.

Dans ce contexte, les conséquences de la sécheresse ont été particulièrement dévastatrices : les zones de repli, plus arrosées, étaient aux mains des cultivateurs; le cheptel sahélien, dont la structure avait été modifiée par les interventions de l'État, était moins armé pour faire face aux aléas climatiques.

Ce diagnostic montre l'importance d'un raisonnement en terme de filière. Il permet la prise en compte intégrée des effets de mesures qui, prises de manière disparate, peuvent amener la disparition d'un système de production adapté à l'exploitation de ressources rares et temporaires.

\section{CONCLUSIONS}

La mise en place d'une filière performante, disposant d'un système de commercialisation efficace et transparent ne constitue pas seulement un lien entre les producteurs et les consommateurs. Elle apporte aussi une contribution positive au développement en stimulant l'activité économique dans le secteur de la production et dans celui des services. 
En outre, la consommation augmente et le producteur tente d'accroître sa production qui, par l'intermédiaire des prix, s'adapte aux besoins du marché.

Bien entendu, il faut que le développement de la production, de la commercialisation et des débouchés soit équilibré. Dans les pays en développement, des contraintes majeures limitent les investissements et les innovations que les populations réclament avec insistance.

Dans ce contexte, la priorité est toujours donnée aux cultures de rente tandis que des expédients commerciaux peu judicieux limitent les possibilités de développement de la production vivrière. La situation est encore aggravée par les lacunes dans l'infrastructure de la plupart des pays en développement et par les entraves que les systèmes inadéquats de commercialisation représentent.

\section{BIBLIOGRAPHIE}

BASLER, A., « Exportations agricoles et déficit alimentaire en Afrique », Economie rurale, 173, 1986, p. 6066.

CASSON, M., « The Theory of Vertical Integration : A Survey and Synthesis, » Journal of Economic Studies, 11,1984 , p. 3-43.

CHATAIGNER, J., « Changements techniques et développement dans les agricultures du tiers monde », Economie rurale, 147,1982 , p. 5-8.

Craplet, C., La Viande de bovins. De l'étable de l'éleveur à l'assiette du consommateur, Paris, Vigot frères, 1965.

EIB, Développement rural intégré, Fascicule $\mathrm{n}^{\circ} 2$ : L'Approche pratique, conclusions des travaux du séminaire organisé à Bordeaux du 16 juin au 11 juillet 1980, Talence, 1980.

FENN, M.C., La Commercialisation du bétail et de la viande, Rome, Organisation des Nations Unies pour l'Alimentation et l'Agriculture, 1979.

FourichoN, J.L., La Filière bovine, Paris, Agra-filières, 1986.

FRENCH, B.C., « The subsector as a Conceptual Framework for Guiding and Conducting Research, » American Journal of Agricultural Economics, 1974, p. 1014-1022.

GIRI, J., Le Sahel demain. Catastrophe ou renaissance, Paris, Karthala, 1983.

HowE, K.S., «Production - Oriented Information Systems for Agricultural Subsectors : Principles and Techniques, » American Journal of Agricultural Economics, 36, 1983, p. 293-301.

JaURon, Y., N. AleXandre, J. LavoIE, L. NADEAU et R. Corbell, éd., Filières de production et développement régional, Office de planification et de développement du Québec, 1977.

KelLoGg, E., R. KodL and P. GARCIA, « The Effects of Agricultural Growth on Agricultural Imports in Developping Countries, American Journal of Agricultural Economics, 68, 1986, p. 1347-1352.

KILMER, R. L., « Vertical Integration in Agricultural and Food Marketing, » American Journal of Agricultural Economics, 68, 1986, p. 1155-1160.

LEDENT, A., éd., Intégration verticale et horizontale en agriculture, Gembloux, Chaire d'économie rurale, Faculté des Sciences agronomiques de l'État, 1986.

LeBAILly, Ph., éd., Étude d'identification relative à la filière de la viande au Tchad, Gembloux, Chaire d'économie rurale, Faculté des Sciences agronomiques de l'État, 1987.

MONFORT, J., « À la recherche des filières de production », Economie et statistique (INSEE), 151, 1983, p. 312.

SEDES, Recueil statistique de la production animale, Paris, Ministère de la Coopération, 1975.

SEKKAT, Kh., "Filières de production : revue de la littérature et comparaison avec la théorie néoclassique ", L'Actualité Économique, 63, 1987, p. 118-142.

SHAFFER, J. D., « Changing Orientations of Marketing Research », American Journal of Agricultural Economics, 50,1968 , p. $1437-1449$.

_ , « On the Concept of Subsector Studies », American Journal of Agricultural Economics, 55, 1973, p. 333335. 
StOFfaEs, C., " Filières et stratégies industrielles », Annales des Mines, 1, 1980, p. 9-19.

TOLlET, R., « Approches méthodologiques de la politique industrielle au travers du concept de filière ", Cahiers Economiques de Bruxelles, 96, p. 496-522.

X, « Sahel et zone soudanienne. L'élevage bovin sans structures », Marchés tropicaux et méditerranéens, 1911, 1982, p. 1611-1621. 